\title{
Mixing in the Solar Tachocline
}

\author{
Allan Sacha Brun \\ JILA, University of Colorado, Boulder, CO 80309-0440, U.S.A. $\&$ \\ DASGAL, Observatoire de Paris, 92195 Meudon, France
}

\begin{abstract}
We conduct numerical simulations of updated solar models including a physical treatment of the tachocline (Spiegel \& Zahn 1992), the rotational transition layer localized at the base of the solar convection zone. We first describe what is the current understanding of this thin shear layer. We then show that we improve substantially the agreement between the models and the observed Sun by taking into account the macroscopic mixing occuring within this region.
\end{abstract}

\section{Introduction}

The presence of a shear layer connecting the differential rotation of the convective zone $(\mathrm{CZ})$ to the solid rotation of the radiative zone is now well established by helioseismic inversions (Corbard et al. 1999). Both its location at $0.691 \pm 0.004$ $R_{\odot}$ and its width of less than $0.05 R_{\odot}$ are well constrained. There are several hydrodynamical or MHD descriptions of this shear layer and its extension but none is definitive. In these models the motions in the tachocline are either turbulent or laminar, involve magnetic fields or are purely hydrodynamical [see Brun \& Zahn (2000) and references therein]. This layer is also presumably the region where the solar global dynamo takes place, making its complete description rather difficult. In our models, we have been using the description of Spiegel \& Zahn (1992) in order to characterize the motions present in the tachocline and thus the amount of mixing. We first recall how we build solar models including a macroscopic diffusivity $D_{T}$. Then we compare our results to the most recent helioseismic data and surface abundance observations for ${ }^{7} \mathrm{Li}$ and ${ }^{9} \mathrm{Be}$.

\section{Implementing macroscopic mixing in solar models}

Macroscopic mixing may be treated in solar models by adding an effective diffusivity $D_{T}$ in the equation for the time evolution of the concentration of chemical species. To establish this coefficient for the tachocline, we use the description by Spiegel \& Zahn (1992) where anisotropic turbulence is responsible for stopping the spread of the layer. This anisotropic diffusion will also interfere with the advective transport of chemicals (Chaboyer \& Zahn 1992). Using these results, Brun, Turck-Chièze and Zahn (1999) have derived an expression for the macroscopic diffusivity $D_{T}$, taking into account the differential rotation profile and 
the tachocline thickness $h$ :

$$
D_{T} \propto \nu_{H}\left(h / r_{b c z}\right)^{2}(\hat{\Omega} / \Omega)^{2},
$$

where, $\nu_{H}$ is the horizontal effective viscosity, $r_{b c z}$ the radius at the base of the convective zone (BCZ) and $\hat{\Omega} / \Omega$ the solar differential rotation of the $\mathrm{CZ}$. In our solar models, we treat $h$ as an adjustable parameter, chosen to agree with the helioseismic determination of the tachocline thickness $h \leq 0.05 R_{\odot}$ (Corbard et al. 1999).

\section{Results: sound speed and abundance of light elements}

Starting from the reference model of Brun, Turck-Chièze and Zahn (1999) built with the CESAM code (Morel 1997), we introduce our coefficient $D_{T}$ in the diffusion equation of chemical species, and we follow the time evolution of the solar model from the pre-main sequence (PMS) until 4.6 Gyr. In Figures 1 and 2 (see Brun \& Zahn 1999 for a more detailed discussion), we compare the results of our reference model, which has only microscopic diffusion, with one (namely $B_{t z}$ ) which includes the macroscopic mixing in the tachocline as explained above, and takes into account its variation in time as the Sun is spun down (Skumanich 1972).

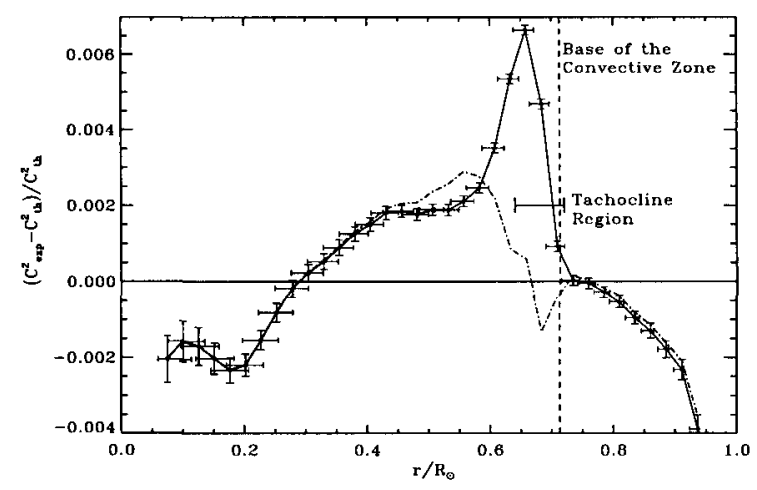

Figure 1. Squared sound speed difference between GOLF+MDI data (error bars) and the reference model (solid curve) and model $B_{t z}$ that incorporates time dependent macroscopic mixing in the tachocline region (dash dot). Also shown are the tachocline extension and the $\mathrm{BCZ}$ (vertical dashed line).

As expected, with macroscopic mixing the composition profile below the convective zone is smoother and it flattens over the depth of the tachocline. Since there is less settling of helium, its photospheric abundance ${ }^{4} \mathrm{He}_{s}=0.2473$ is slightly higher, in better agreement with the helioseismic determined value. This indicates that the purely microscopic diffusive model is overestimating the effect of gravitational settling on chemical species during the Sun's evolution.

The effect on the sound speed is displayed in Figure 1. When the macroscopic transport is neglected (solid curve with error bars), the squared sound 
speed difference reveals a peak just below the CZ, coinciding with the tachocline. Macroscopic diffusion acts to reduce this peak, but when one recalibrates the model to yield the present abundance of heavy elements $(Z / X=0.0245 \pm 0.002)$, the effect is rather minor. On the other hand, if the heavy elements are left free to adjust within the observational uncertainties, the peak is completely removed, leaving only a broad bump positioned at $0.6 R_{\odot}$, which presumably is due to another cause (dash dotted curve).

The two light elements ${ }^{7} \mathrm{Li}$ and ${ }^{9} \mathrm{Be}$ are extremely sensitive to mixing processes occuring in stars because their nuclear burning temperatures are rather low (respectively $2.5 \times 10^{6}$ and $3.2 \times 10^{6} \mathrm{~K}$ ). The new observational constraints can only be satisfied if those chemical species are mixed in a rather thin layer below the $\mathrm{CZ}$ in order to preserve ${ }^{9} \mathrm{Be}$, which is very little depleted according to Balachandran and Bell (1998). This is the case with our tachocline model, which extends only $5 \%$ below the base of the convective zone.

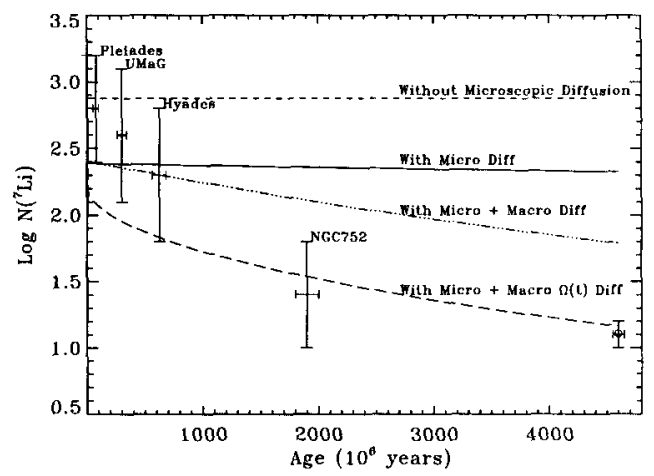

Figure 2. Depletion of ${ }^{7} \mathrm{Li}$ versus evolution time for several solar models: no microscopic diffusion (dash), with microscopic diffusion (solid curve) and with mixing within the tachocline: no time dependence (dash three dots), and with a time dependence (i.e model $B_{t z}$, with initial heavy element abundance $\mathrm{Z}_{0}=\mathrm{Z}_{0}^{\text {ref }}=0.01959$ ) (long dash). We superimposed on the theoretical curves the open cluster observations (see Brun et al. 1999 and references therein).

In Figure 2 we show the resulting lithium depletion occurring during the Sun's evolution for different models, including one without any diffusive processes. Clearly only the diffusive models including macroscopic mixing in the tachocline yield a substantial depletion during main sequence evolution, in agreement with the observations (superimposed with their inherent dispersion on the theoretical curves). But it is important to note that the thickness of the tachocline and the strength of mixing have been larger in the past (see Brun et al. 1999), when the Sun was rotating faster. When this time dependence is included in the models (as in model $B_{t z}$ ), the lithium is burned more effectively and the models present a reasonable value of the solar ${ }^{7} \mathrm{Li}$ depletion $(\sim 100)$. However, the lithium depletion during PMS is probably overestimated due to the crude spin-down law we have adopted; we have also neglected the influence of the possibly strong magnetic field present in the early phases of the Sun. 
In conclusion, this study demonstrates the crucial role of the thin tachocline layer below the $\mathrm{CZ}$ in governing the photospheric abundance of the three elements ${ }^{4} \mathrm{He},{ }^{7} \mathrm{Li},{ }^{9} \mathrm{Be}$, and it stresses the importance of describing accurately the macroscopic mixing processes which are operating there.

Acknowledgments. I would like to thank S. Turck-Chièze and J.-P. Zahn for their support and comments on this work and J. Toomre for useful discussion. This work was partly supported by NASA through grants NAG5-2256 and NAG5-8133.

\section{References}

Balachandran, S. \& Bell, R. A. 1998, Nature, 392, 791

Brun, A. S., Turck-Chièze, S. \& Zahn, J.-P. 1999, ApJ, 525, 1032

Brun, A. S. \& Zahn, J.-P. 2000, Ann. N.Y. Acad. Sci., 898, 113

Chaboyer, B. \& Zahn, J.-P. 1992, A\&A, 253, 173

Corbard, T. et al. 1999, A\&A, 344, 696

Morel, P. 1997, A\&AS, 124, 597

Skumanich, A. 1972, ApJ, 171, 565

Spiegel, E. A. \& Zahn, J.-P. 1992, A\&A, 265, 106 